\title{
Bilateral Cavernous Sinus Thrombosis in Lemierre's Syndrome
}

\author{
Adrian Budhram, Basavaraj Shettar, Donald H. Lee, Michael Silverman, \\ Kaveri Gupta
}

Keywords: Meningitis, neuroinfectious disease, thrombosis, headache, magnetic resonance imaging, neuroimaging, Lemierre's syndrome

doi:10.1017/cjn.2016.438

Can J Neurol Sci. 2017; 44: 424-426

A 51-year-old male presented with one week of right-sided partial ophthalmoplegia and ptosis, as well as a four-month history of headache after right upper tooth extraction. His medical history included type 2 diabetes mellitus, and he was a 25-packyear smoker. On examination, he was afebrile, and other than his right-sided extraocular findings, neurological and general systems examinations were unremarkable. Gadolinium-enhanced magnetic resonance imaging (MRI) of the head showed focal, predominantly right-sided anterior pachymeningeal enhancement (Figure 1). Lumbar puncture revealed 541 neutrophilpredominant white blood cells, but cerebrospinal fluid and blood cultures were negative. Computed tomography of the thorax/ abdomen/pelvis revealed bilateral lung nodules, concerning for malignancy given his smoking history. Nodule biopsy, however, showed fibrinopurulent exudate suggestive of an infectious or inflammatory process.

Eight days after his initial MRI, the patient acutely developed near-complete bilateral ophthalmoplegia and ptosis. Repeat gadolinium-enhanced MRI showed interval development of bilateral cavernous sinus enhancement defects and diffusion restriction, concerning for bilateral cavernous sinus thrombosis. Predominantly left-sided sigmoid sinus and jugular bulb irregularities were seen, also compatible with thrombosis (Figure 2). He remained afebrile, but two weeks after initial presentation, $2 / 2$ repeat blood cultures grew multiple anaerobes, including Actinomyces meyeri, Parvimonas micra and Fusobacterium species. This confirmed Lemierre's syndrome, an illness characterized by internal jugular vein thrombophlebitis with anaerobic bacteremia, classically Fusobacterium necrophorum. ${ }^{1,2}$ Our patient acutely developed clinical and radiographic evidence of bilateral cavernous sinus thrombosis, a rare complication of Lemierre's syndrome that may be life-threatening if not promptly recognized and treated. He also had focal pachymeningitis and septic lung foci, illustrating the diverse potential clinical manifestations of this disease. On thorough oral examination, the dentistry service found and extracted a residual root, suggesting an odontogenic source. ${ }^{2}$ Marked clinico-radiographic improvement occurred after six weeks of meropenem 2 grams IV q8h, metronidazole $500 \mathrm{mg}$ PO TID, and rivaroxaban $20 \mathrm{mg}$ PO daily, emphasizing the importance of accurate diagnosis and treatment.

\section{Disclosures}

Adrian Budhram, Basavaraj Shettar, Donald H. Lee, Michael Silverman, and Kaveri Gupta hereby declare that they have nothing to disclose.

\section{STATEMENT OF AUTHORSHIP}

Adrian Budhram conducted patient examinations and clinical follow-up, drafted the manuscript and drew the figures. Basavaraj Shettar conducted patient examinations and performed critical revision of the manuscript for intellectual content. Donald $H$. Lee performed examination of patient radiographs and critical revision of the neuroimaging content. Michael Silverman conducted patient examinations and performed critical revision ofthe manuscript for intellectual content. Kaveri Gupta conducted patient examinations and clinical follow-up, and performed critical revision of the manuscript for intellectual content.

\section{REFERENCES}

1. Lemierre A. On certain septicaemias due to anaerobic organisms. Lancet. 1936;227(5874):701-3.

2. Noy D, Rachmiel A, Levy-Faber D, Emodi O. Lemierre's syndrome from odontogenic infection: review of the literature and case description. Ann Maxillofac Surg. 2015;5(2):219-25. Available at: https://www.ncbi.nlm.nih.gov/pmc/articles/PMC4772564/.

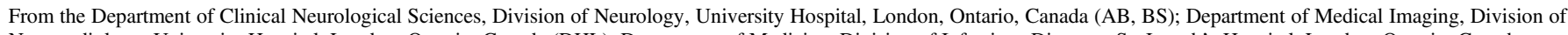
Neuroradiology, University Hospital, London, Ontario, Canada (DHL); Department of Medicine, Division of Infectious Diseases, St. Joseph's Hospital, London, Ontario, Canada (MS, KG).

Received August 6, 2016. Final Revisions Submitted SePtember 4, 2016. Date of acceptance SePtember $27,2016$.

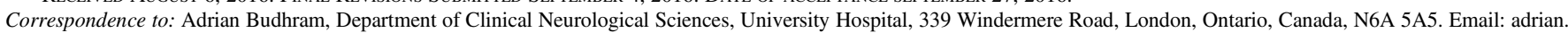
budhram@medportal.ca. 

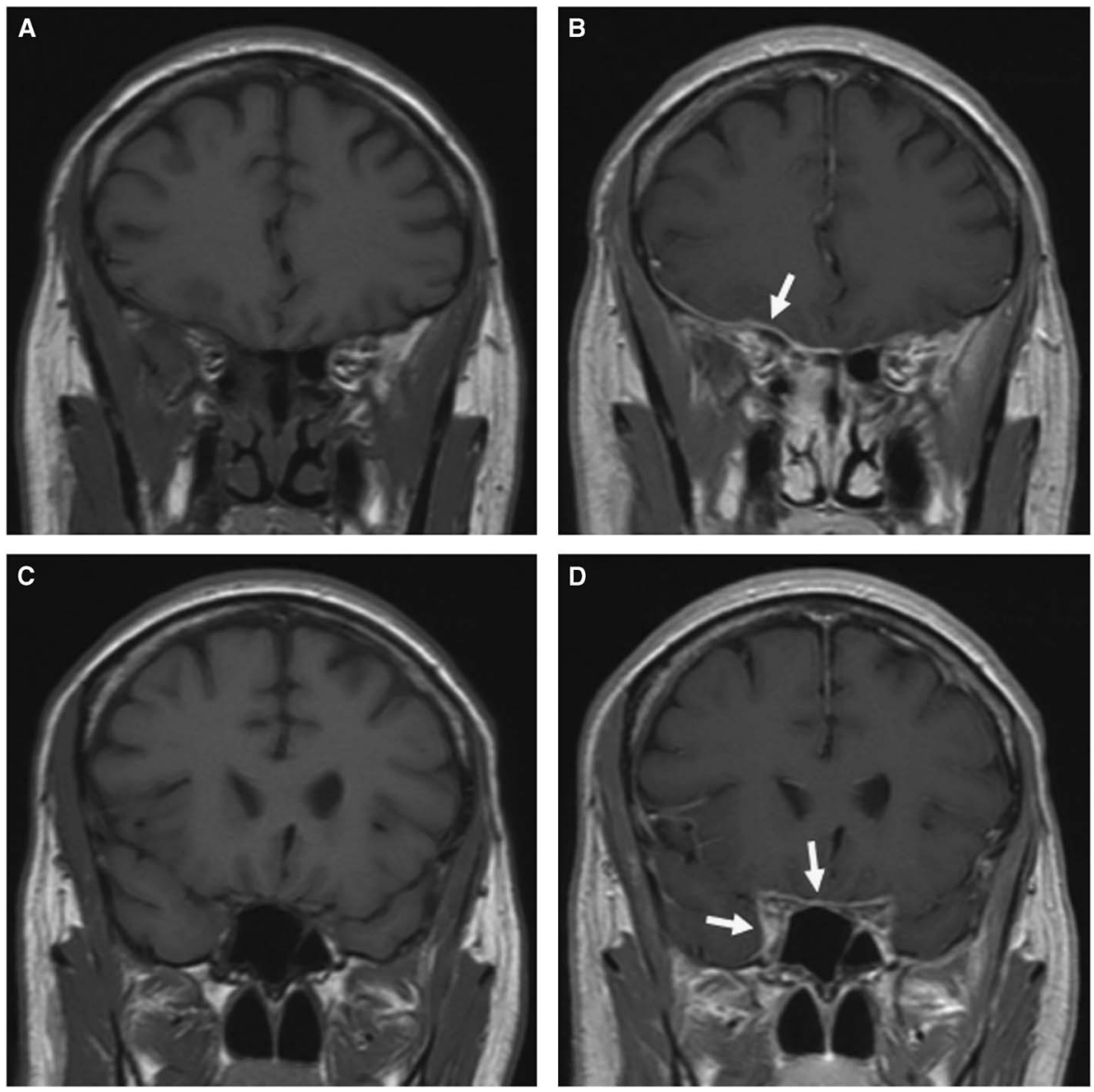

Figure 1: Focal pachymeningeal enhancement on initial gadolinium-enhanced MRI head. Coronal T1-weighted pre-gadolinium (panels $A$ and $C$ ) and post-gadolinium images showing pachymeningeal enhancement of the right basal frontal lobe (panel B, arrow), as well as the planum sphenoidale and anterior right cavernous sinus (panel $D$, arrows). 

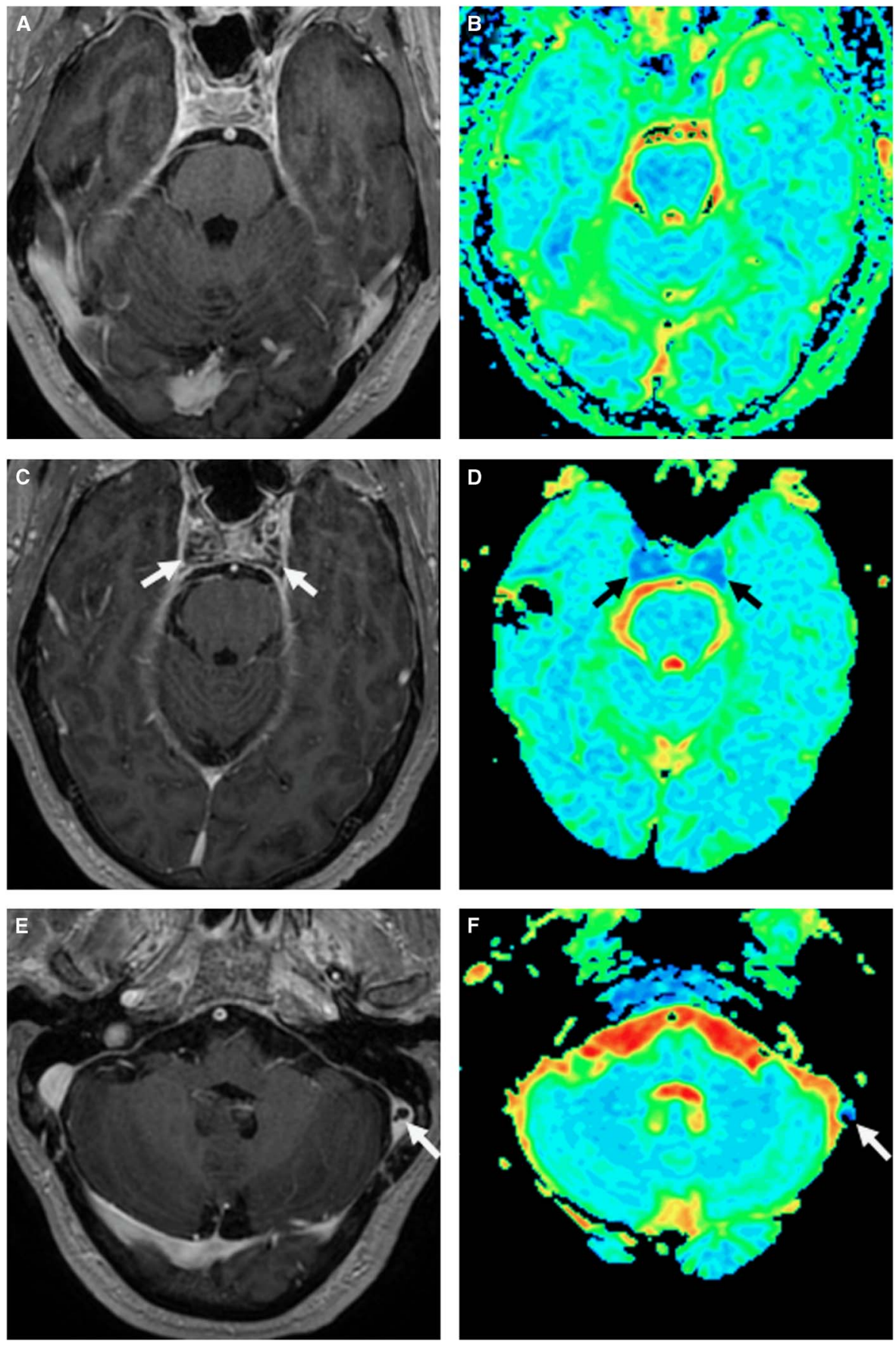

Figure 2: Cavernous sinus and sigmoid sinus thrombosis on repeat gadolinium-enhanced MRI head. Axial T1-weighted post-gadolinium (panel A) and axial colour scale apparent diffusion coefficient (ADC) map (panel B) imaging of cavernous sinuses on initial MRI is unremarkable. Repeat MRI eight days later shows interval subtle cavernous sinus enlargement and postgadolinium filling defects on axial T1-weighted post-gadolinium images (panel C, arrows), as well as diffusion restriction of the cavernous sinuses bilaterally on axial colour scale ADC map (panel $D$, arrows). An irregular left sigmoid sinus lucency on axial T1-weighted post-gadolinium imaging (panel E, arrow) with trace diffusion restriction on axial colour scale ADC map (panel F, arrow) is also seen. 